\title{
Removal of the Power Line Interference from ECG Signal Using Different Adaptive Filter Algorithms and Cubic Spline Interpolation for Missing Data Points of ECG in Telecardiology System
}

\author{
Md Salah Uddin Farid, Shekh Md Mahmudul Islam* \\ Department of Electrical and Electronic Engineering, University of Dhaka, Bangladesh
}

Submission: December 18, 2017; Published: March 13, 2018

*Corresponding author: Md Mahmudul Islam, Department of Electrical and Electronic Engineering, University of Dhaka, Dhaka, Bangladesh, Email: mahmud@du.ac.bd

\begin{abstract}
Maintaining one's health is a fundamental human right although one billion people do not have access to quality healthcare services. Telemedicine can help medical facilities reach their previously inaccessible target community. The Telecardiology system designed and implemented in this research work is based on the use of local market electronics. In this research work we tested three algorithms named as LMS (Least Mean Square), NLMS (Normalized Mean Square), and RLS (Recursive Least Square). We have used $250 \mathrm{mV}$ amplitude ECG signal from MITBIH database, $25 \mathrm{mV}$ ( $10 \%$ of original ECG signal) of random noise, white Gaussian noise and $100 \mathrm{mV}$ ( $40 \%$ of original ECG signal) of $50 \mathrm{~Hz}$ power signal noise is added with ECG signal in different combinations and Adaptive filter with three different algorithms have been used to reduce the noise that is added during transmission through the telemedicine system. Normalized mean square error was calculated and our MATLAB simulation results suggest that RLS performs better than other two algorithms to reduce the noise from ECG. During analog transmission of ECG signal through existing Telecommunication network some data points may be lost and we have theoretically used Cubic Spline interpolation to regain missing data points. We have taken 5000 data points of ECG Signal from MIT -BIH database. The normalized mean square error was calculated for regaining missing data points of the ECG signal and it was very less in all the conditions. Cubic Spline Interpolation function on MATLAB platform could be a good solution for regaining missing data points of original ECG signal sent through our proposed Telecardiology system but practically it may not be efficient one.
\end{abstract}

Keywords: Telemedicine; Power line interference (PLI); ECG; Adaptive filter; LMS; NLMS; RLS

Abbreviations: EMG: Electromyography; ECG: Electrocardiogram; EEG: Electroencephalogram; NLMS: Normalized Least Mean Square; RLS: Recursive Least Square; SD: Storage Card

\section{Introduction}

The ECG signal measured with an electrocardiograph, is a biomedical electrical signal occurring on the surface of the body related to the contraction and relaxation of the heart. This signal represents an extremely important measure for doctors as it provides vital information about a patient cardiac condition and general health. Generally the frequency band of the ECG signal is 0.05 to $100 \mathrm{~Hz}$ [1]. Inside the heart there is a specialized electrical conduction system that ensures the heart to relax and contracts in a coordinated and effective fashion. ECG recordings may have common artifacts with noise caused by factors such as power line interference, external electromagnetic field, random body movements, other biomedical signals and respiration. Different types of digital filters may be used to remove signal components from unwanted frequency ranges [2].
The Power line interference $50 / 60 \mathrm{~Hz}$ is the source of interference in bio potential measurement and it corrupt the biomedical signal's recordings such as Electrocardiogram (ECG), Electroencephalogram (EEG) and Electromyography (EMG) which are extremely important for the diagnosis of patients. It is hard to find out the problem because the frequency range of ECG signal is nearly same as the frequency of power line interference. The QRS complex is a waveform which is most important in all ECG's waveforms and it comes into view in usual and unusual signals in ECG [3]. As it is difficult to apply filters with fixed coefficients to reduce biomedical signal noises because human behaviour is not exact depending on time, an adaptive filtering technique is required to overcome the problem. Adaptive filer is designed using different algorithms such as least mean square 
(LMS), Normalized least mean square (NLMS), Recursive least square (RLS) [4]. Least square algorithms aims at minimization of the sum of the squares of the difference between the desired signal and model filter output when new samples of the incoming signals are received at every iteration, the solution for the least square problem can be computed in recursive form resulting in the recursive least square algorithm. The goal for ECG signal enhancement is to separate the valid signal components from the undesired artifacts so as to present an ECG that facilitates an easy and accurate interpretation.

The basic idea for adaptive filter is to predict the amount of noise in the primary signal and then subtract noise from it. In this research work a Telecardiology system has been designed and implemented using instrumentation amplifier, band pass filter and Arduino interfacing between Smartphone and Arduino board. First of all raw ECG signal has been amplified and filtered by Band pass filter. Analog signal has been digitized using Arduino board and then interfacing between Arduino board and smart phone has been implemented and Digitized value of analog signal has been sent from Arduino board to smart phone and digitized value of analog signal has been stored in SD storage card of smart phone. Using Bluetooth or existing Telecommunication Network. As sinusoidal signals are known to be corrupted during transmission it is expected that similarly an ECG signal will be corrupted.

We have therefore designed an adaptive filter with three different algorithms and simulated in MATLAB platform to compare the performance of denoising of ECG signal. During transmission of ECG signals through existing Telecommunication networks some data pints may be lost. In this research work we have used cubic spline interpolation to regain missing data points. If more data points are missing then reconstruction of an ECG signal becomes impossible and doctor can not accurately interpret a patient's ECG in an efficient manner. Cubic spline interpolation has been implemented for various missing data points of original ECG signal taken from MIT-BIH database. The normalized mean square error of cubic spline interpolation was very low. Cubic Spline interpolation in Matlab platform could be a better solution for regaining missing data points of ECG signal theoretically.

\section{Related Works and Literature Review}

The extraction of high-resolution ECG signals from recordings contaminated with system noise is an important issue to investigate in Telecardiology system. The goal for ECG signal enhancement is to separate the valid signal components from the undesired artifacts, so as to present an ECG that facilitates easy and accurate interpretation.

The work of this research paper is the development of our previous research work "Denoising ECG Signal using Different Adaptive Filter Algorithms and Cubic Spline Interpolation for Missing data points of ECG in Telecardiology System" [5]. Many approaches have been reported in the literature to address ECG enhancement using adaptive filters [6-9], which permit to detect time varying potentials and to track the dynamic variations of the signals. In Md. Maniruzzaman et al $[7,10,11]$ proposed wavelet packet transform, Least-Mean-Square (LMS) normalized least-mean-square (NLMS) and recursive-least-square (RLS), and the results are compared with a conventional notch filter both in time and frequency domain. In these papers, power line interference noise is denoised by NLMS or LMS or RLS algorithms and performed by MTLAB or LABVIEW. But in our research work we have developed our previous work. We denoised ECG signal by removing power line interference, Random noise and White Gaussian noise. In our previous research paper [12] we denoised ECG signal from random noise and white Gaussian noise. In our present research work we have added power line interference with Pure ECG signal individually and added in mixed of power line interference, random noise and white Gaussian noise in different combinations. Finally in our research work we have used cubic spline interpolation for regaining missing data points of ECG signal sent through telecommunication network.

There are certain clinical applications of ECG signal processing that require adaptive filters with large number of taps. In such applications the conventional LMS algorithm is computationally expensive to implement The LMS algorithm and NLMS (normalized LMS) algorithm require few computations, and are, therefore, widely applied for acoustic echo cancellers. However, there is a strong need to improve the convergence speed of the LMS and NLMS algorithms. The RLS (recursive least-squares) algorithm, whose convergence does not depend on the input signal, is the fastest of all conventional adaptive algorithms. The major drawback of the RLS algorithm is its large computational cost. However, fast (small computational cost) RLS algorithms have been studied recently. In this paper we aim to obtain a comparative study of faster algorithm by incorporating knowledge of the room impulse response into the RLS algorithm. Unlike the NLMS and projection algorithms, the RLS algorithm does not have a scalar step size.

Therefore, the variation characteristics of an ECG signal cannot be reflected directly in the RLS algorithm. Here, we study the RLS algorithm from the viewpoint of the adaptive filter because

a. The RLS algorithm can be regarded as a special version of the adaptive filter and

b. Each parameter of the adaptive filter has physical meaning.

Computer simulations demonstrate that this algorithm converges twice as fast as the conventional algorithm. These characteristics may plays a vital role in biotelemetry, where extraction of noise free ECG signal for efficient diagnosis and fast computations, high data transfer rate are needed to avoid overlapping of pulses and to resolve ambiguities. To the best 
of our knowledge, transform domain has not been considered previously within the context of filtering artifacts in ECG signals.

In this paper we compare the performances of LMS, NLMS and RLS algorithms to remove the artifacts from ECG. This algorithm enjoys less computational complexity and good filtering capability. To study the performance of the algorithms to effectively remove the noise from the ECG signal, we carried out simulations on MIT-BIH database. During transmission of ECG signal through existing Telecommunication network it may be corrupted or some data points may be lost. Linear Spline interpolation was popular method for regaining missing data points of ECG signal [13]. Cubic Spline interpolation has gained popularity very recently [6]. In our previous research work we used cubic spline interpolation. The development of our previous research work i.e., in the present research work, in this research paper we have also used cubic spline interpolation for regaining missing data points of ECG signal sent through telecommunication network.

\section{Adaptive Filter Algorithms}

In this research work a Telecardiology system has been implemented and proposed for sending ECG signal through smart phone (Figure 1).

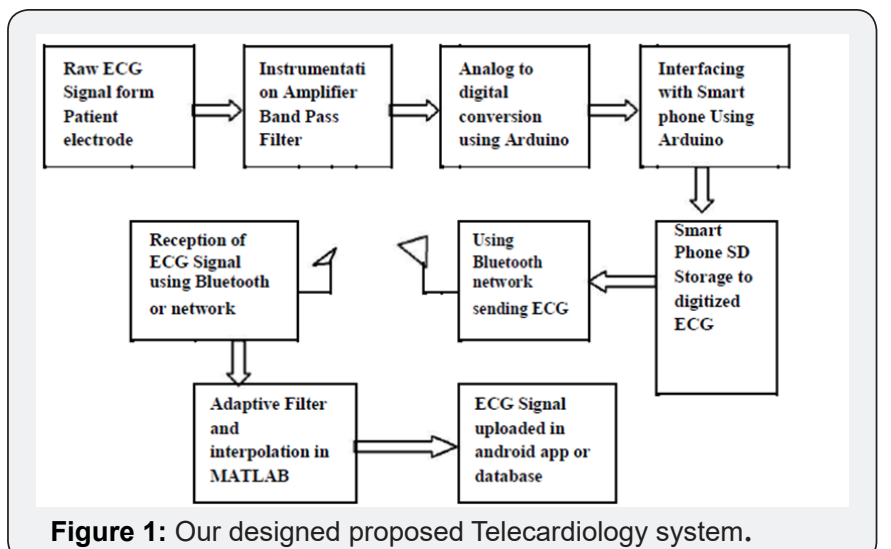

The raw ECG signal will be taken from patient electrode and passed through instrumentation amplifier and band pass filter to amplify the signal and to reduce the noise coming from electrodes. Then that amplified and filtered analog ECG signal will be converted into digital signal by using Arduino AVR microcontroller based system. Then that digital value of ECG signal will be sent to smart phone by using Arduino interfacing with smart phone and digitized signal values will be sent to smart phone SD card. After that digital value will be sent to another smart phone by using Bluetooth technology. Digitized ECG value will be received to smart phone via Bluetooth .During transmission of ECG signal through Telecommunication network it may be corrupted by random noise or white Gaussian noise of the network. Adaptive filter using different algorithms have been used to reduce noise of the transmitted ECG signal. A MATLAB coding has been done to reduce the noise of the ECG signal and for reducing noise of digitized ECG signal, transmitted noisy ECG signal needs to be loaded in MATLAB and then it is filtered suing adaptive filter with different algorithms and performances of different algorithms are measured based on their de-noising capabilities. During transmission of ECG signal some data points may be missing and MATLAB spline interpolation algorithm will get them back so that ECG signal can be transmitted reliably (Figure 2).

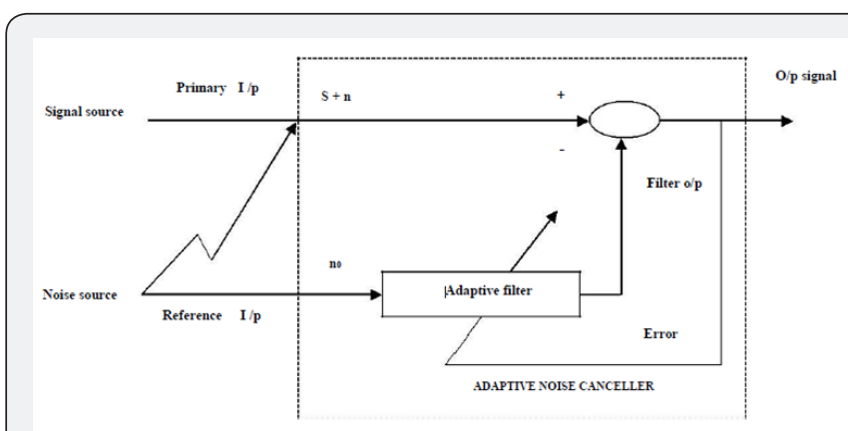

Figure 2: Adaptive Noise Canceller (ANC).

Least Mean Square (LMS), Normalized Mean Square Algorithm (NLMS) and Recursive Least Square Algorithm (RLS) has been designed and implemented for denoising ECG signal in MATLAB platform [4,12-14]. Cubic Spline Interpolation has been used for regaining missing data points of ECG signal during transmission through existing Telecommunication network. The normalized mean square error was calculated for regaining missing data points of ECG signal and it was very less and so Cubic spline interpolation could be a better solution in MATLAB platform for regaining missing data points of ECG signal.

\section{Result}

In this work we have taken pure ECG signal from MIT-BIH database. The amplitude of our taken ECG signal was $250 \mathrm{mV}$ which is amplified from. $5 \mathrm{mV}$ ( $2 \%$ of original ECG signal), $10 \mathrm{mV}$ ( $4 \%$ of original ECG) $15 \mathrm{mV}$ ( $6 \%$ of original ECG), $20 \mathrm{mV}$ ( $8 \%$ of original ECG signal) and $25 \mathrm{mV}$ (10\% of original ECG signal) of random noise and white Gaussian noise is added with ECG signal. Three different algorithms of Adaptive filter were implemented and tested their performances of denosing ECG signal. We have taken ECG signal with $250 \mathrm{mV}$ amplitude and 5000 samples were taken from MIT-BIH database (Figure 3). In our simulation work we have denoised $100 \mathrm{mV}$ of $50 \mathrm{~Hz}$ power signal noise.

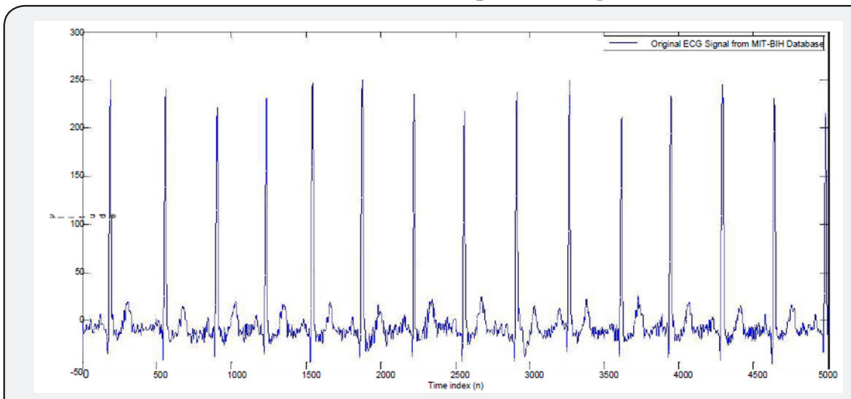

Figure 3: Pure ECG signal taken from MIT-BIH database. 
Least Mean square (LMS) Algorithms (Figure 4-7)
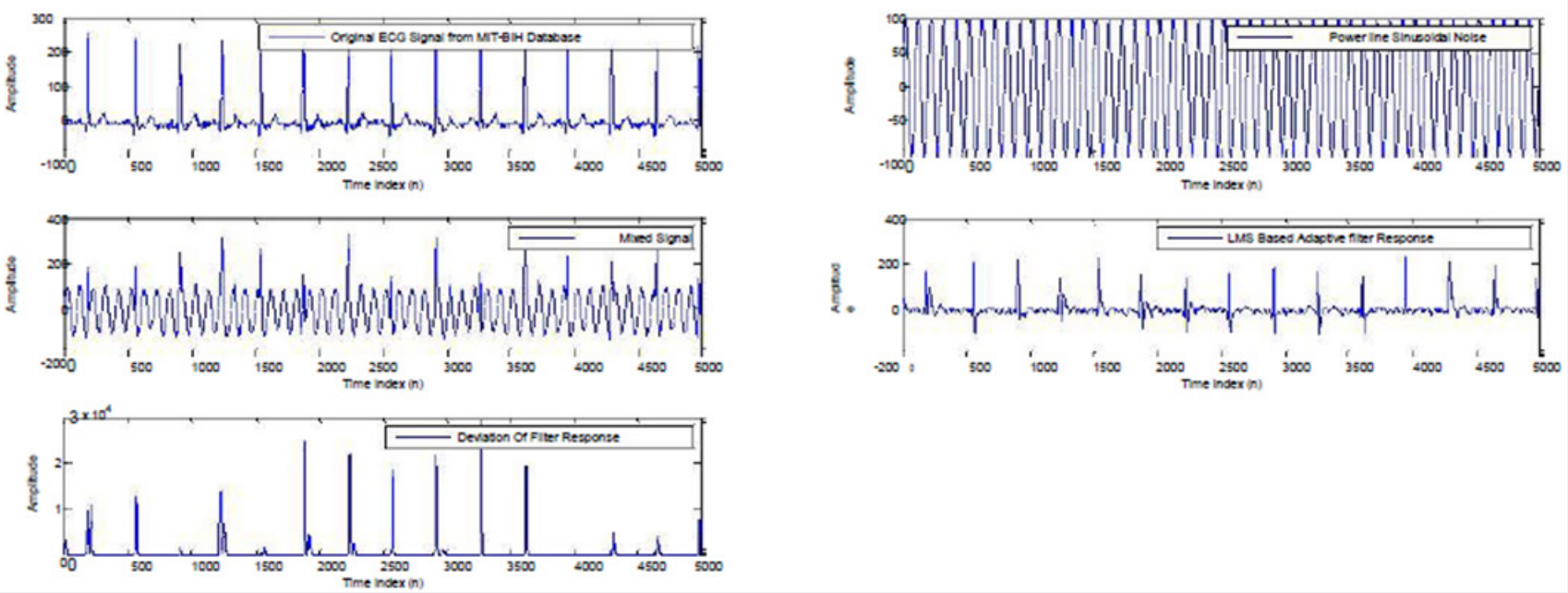

Figure 4: (a) Pure ECG signal taken from MIT-BIH Database (b) $50 \mathrm{~Hz}$ power line sinusoidal noise with average amplitude $100 \mathrm{mV}$ (c) $50 \mathrm{~Hz}$ power line sinusoidal noise mixed with pure ECG signal (d) LMS based Adaptive Filter response (e) Square Deviation of LMS based adaptive filter.

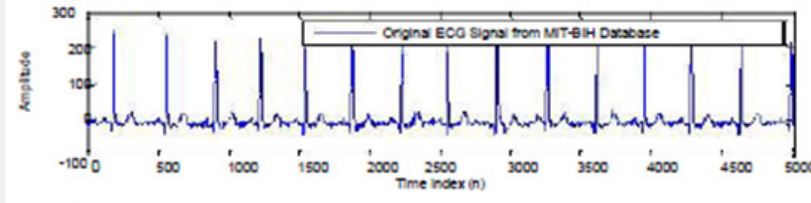

2004

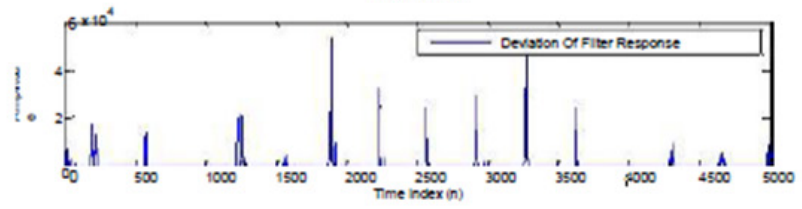

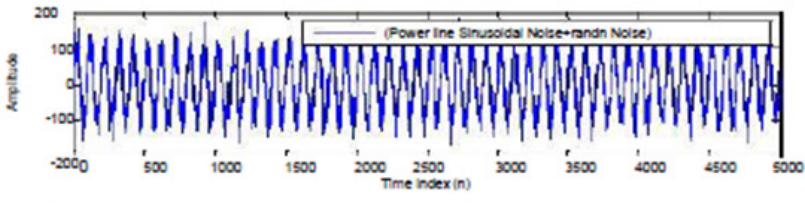

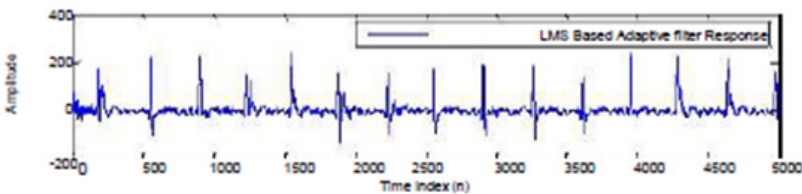

Figure 5: (a) Pure ECG signal taken from MIT-BIH Database (b) $50 \mathrm{~Hz}$ power line sinusoidal noise with average amplitude $100 \mathrm{mV}+\mathrm{Random}$ Noise with average amplitude $25 \mathrm{mV}$ (c) $50 \mathrm{~Hz}$ power line sinusoidal noise \& Random Noise are mixed with pure ECG signal (d) LMS based Adaptive Filter response (e) Square Deviation of LMS based adaptive filter.

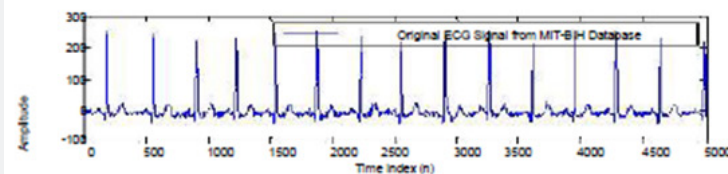

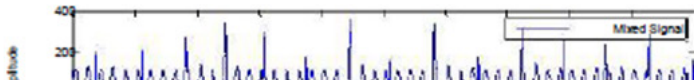



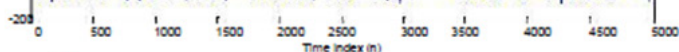

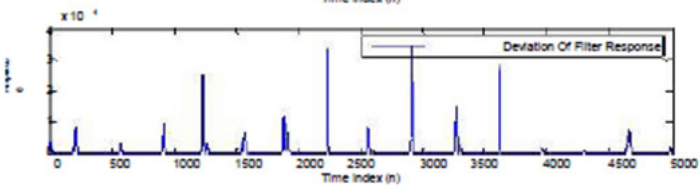

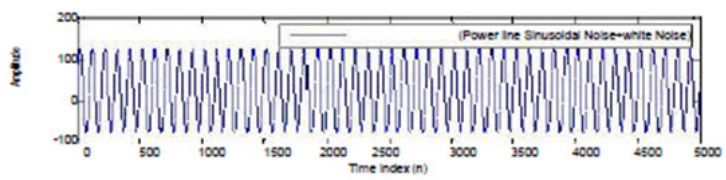



Figure 6: (a) Pure ECG signal taken from MIT-BIH Database (b) $50 \mathrm{~Hz}$ power line sinusoidal noise with average amplitude 100 mV+ Gaussian Noise with average amplitude $25 \mathrm{mV}$ (c) $50 \mathrm{~Hz}$ power line sinusoidal noise \& Gaussian Noise are mixed with pure ECG signal (d) LMS based Adaptive Filter response (e) Square Deviation of LMS based adaptive filter. 

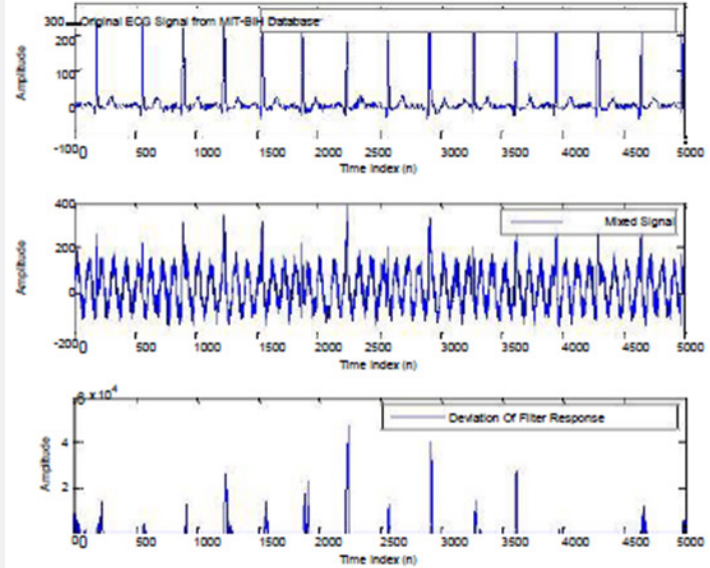

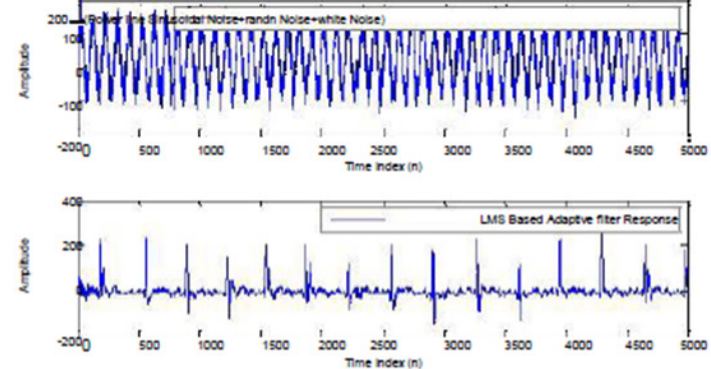
with average amplitude $25 \mathrm{mV}+$ Random Noise with average amplitude $25 \mathrm{mV}$ (c) $50 \mathrm{~Hz}$ power signal noise, Gaussian Noise and random Noise are mixed with pure ECG signal (d) LMS based Adaptive Filter response (e) Square Deviation of LMS based adaptive filter.

Normalized Mean Square (NLMS) Algorithms (Figure 8-11)
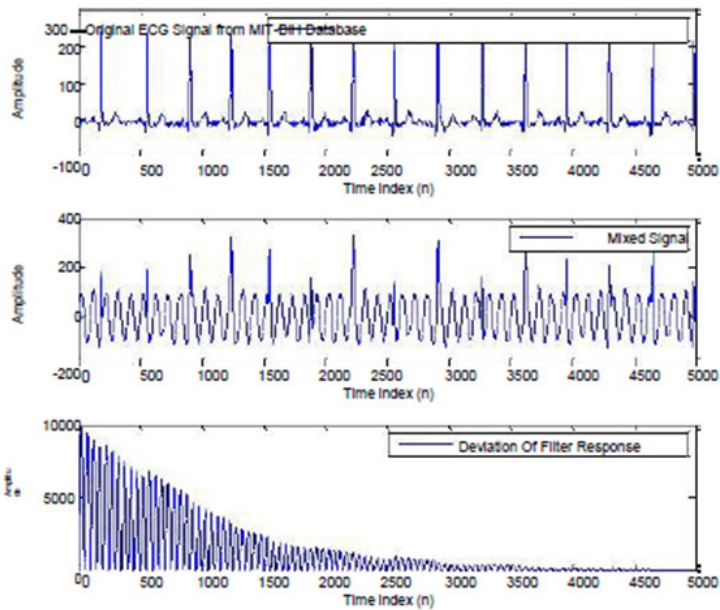
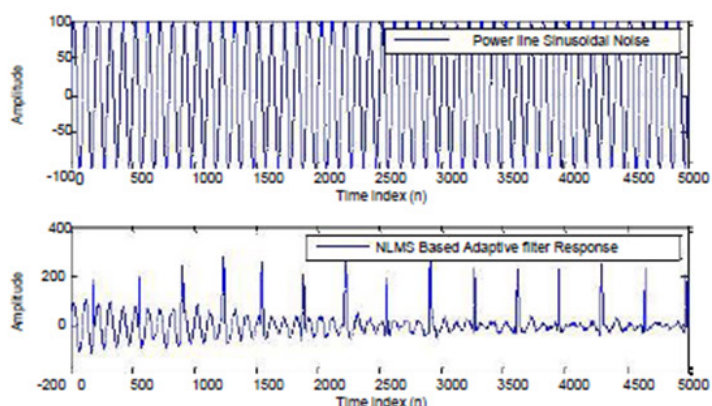

Figure 8: (a) Pure ECG signal taken from MIT-BIH Database (b) $50 \mathrm{~Hz}$ power signal noise with average amplitude $100 \mathrm{mV}$ (c) $50 \mathrm{~Hz}$ power signal noise mixed with pure ECG signal (d) NLMS based Adaptive Filter response (e) Square Deviation of NLMS based adaptive filter.

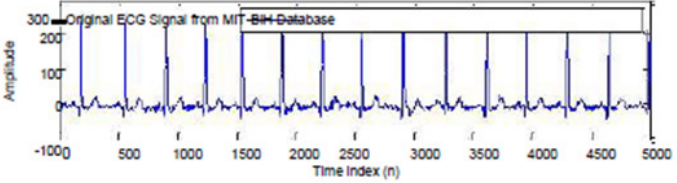

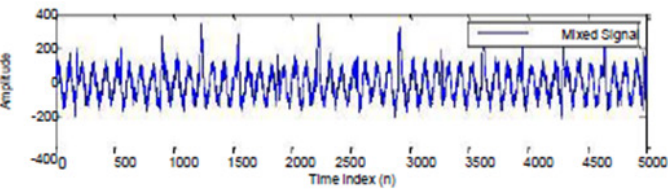

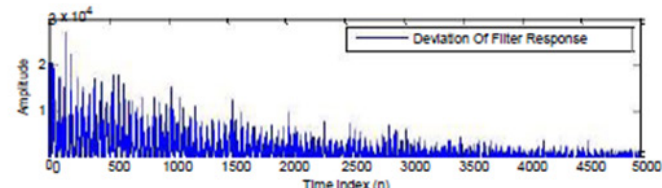

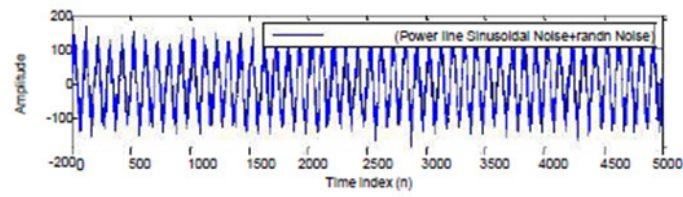

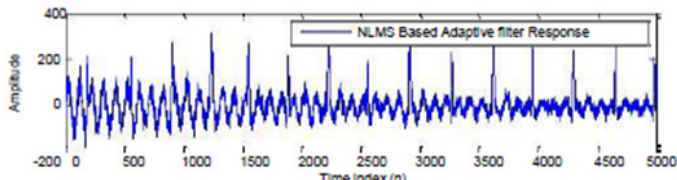

Figure 9: (a) Pure ECG signal taken from MIT-BIH Database (b) $50 \mathrm{~Hz}$ power signal noise with average amplitude $100 \mathrm{mV}+\mathrm{Random}$ Noise with average amplitude $25 \mathrm{mV}$ (c) $50 \mathrm{~Hz}$ power signal noise \& Random Noise are mixed with pure ECG signal (d) NLMS based Adaptive Filter response (e) Square Deviation of NLMS based adaptive filter. 

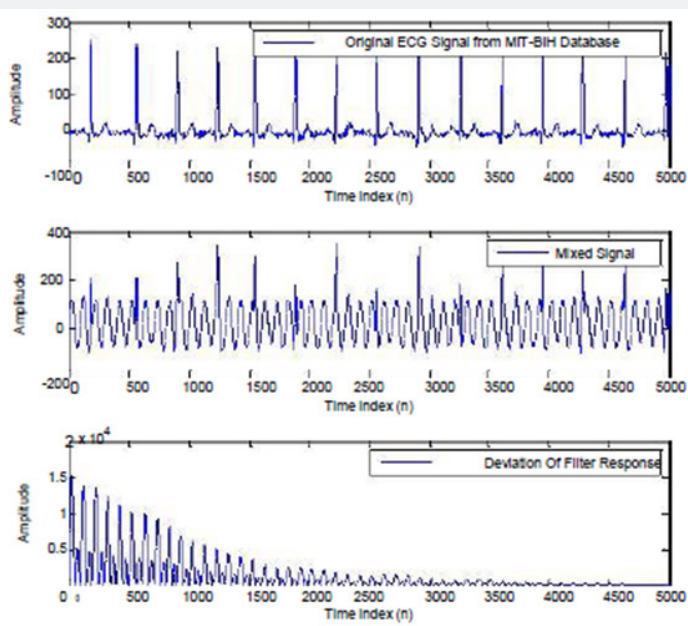
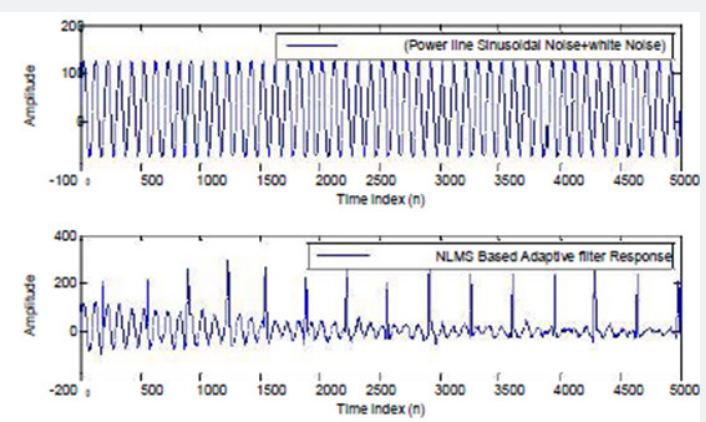

Figure 10: (a) Pure ECG signal taken from MIT-BIH Database (b) $50 \mathrm{~Hz}$ power signal noise with average amplitude $100 \mathrm{mV}+\mathrm{Gaussian}$ Noise with average amplitude $25 \mathrm{mV}$ (c) $50 \mathrm{~Hz}$ power signal noise \& Gaussian Noise are mixed with pure ECG signal (d) NLMS based Adaptive Filter response (e) Square Deviation of NLMS based adaptive filter.
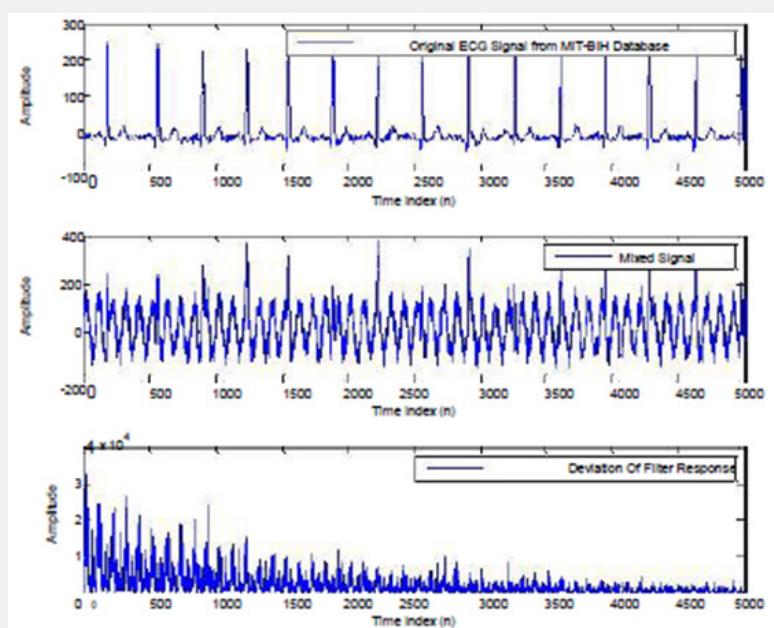
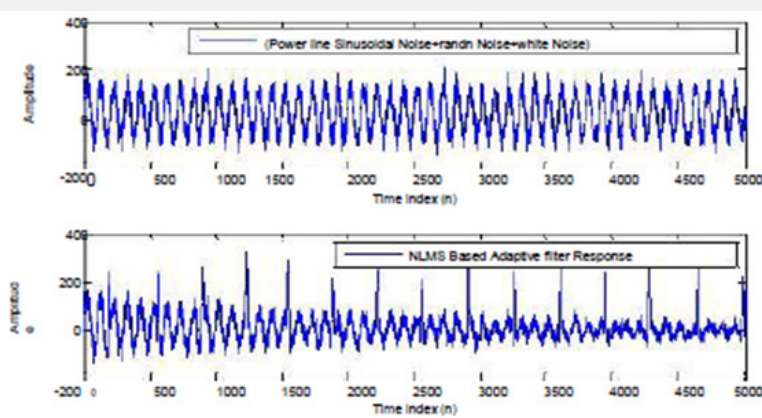

Figure 11: (a) Pure ECG signal taken from MIT-BIH Database (b) $50 \mathrm{~Hz}$ power signal noise with average amplitude $100 \mathrm{mV}+\mathrm{Gaussian}$ Noise with average amplitude $25 \mathrm{mV}+$ Random Noise with average amplitude $25 \mathrm{mV}$ (c) $50 \mathrm{~Hz}$ power signal noise, Gaussian Noise and random Noise are mixed with pure ECG signal (d) NLMS based Adaptive Filter response (e) Square Deviation of NLMS based adaptive filter.

Recursive Least Square (RLS) Algorithms (Figure 12-14)
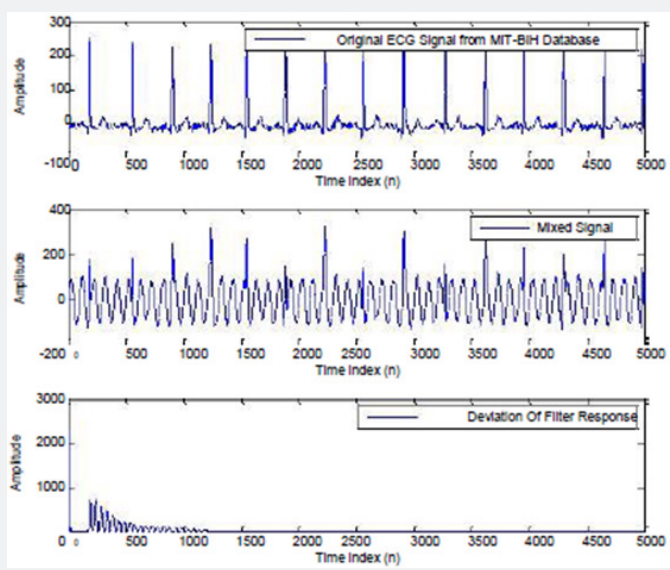
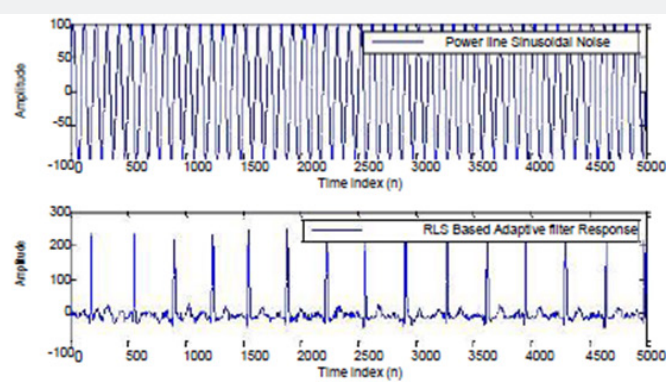

Figure 12: (a) Pure ECG signal taken from MIT-BIH Database (b) $50 \mathrm{~Hz}$ power line sinusoidal noise with average amplitude $100 \mathrm{mV}+$ Random Noise with average amplitude $25 \mathrm{mV}$ (c) $50 \mathrm{~Hz}$ power signal noise \& Random Noise are mixed with pure ECG signal (d) RLS based Adaptive Filter response (e) Square Deviation of RLS based adaptive filter. 

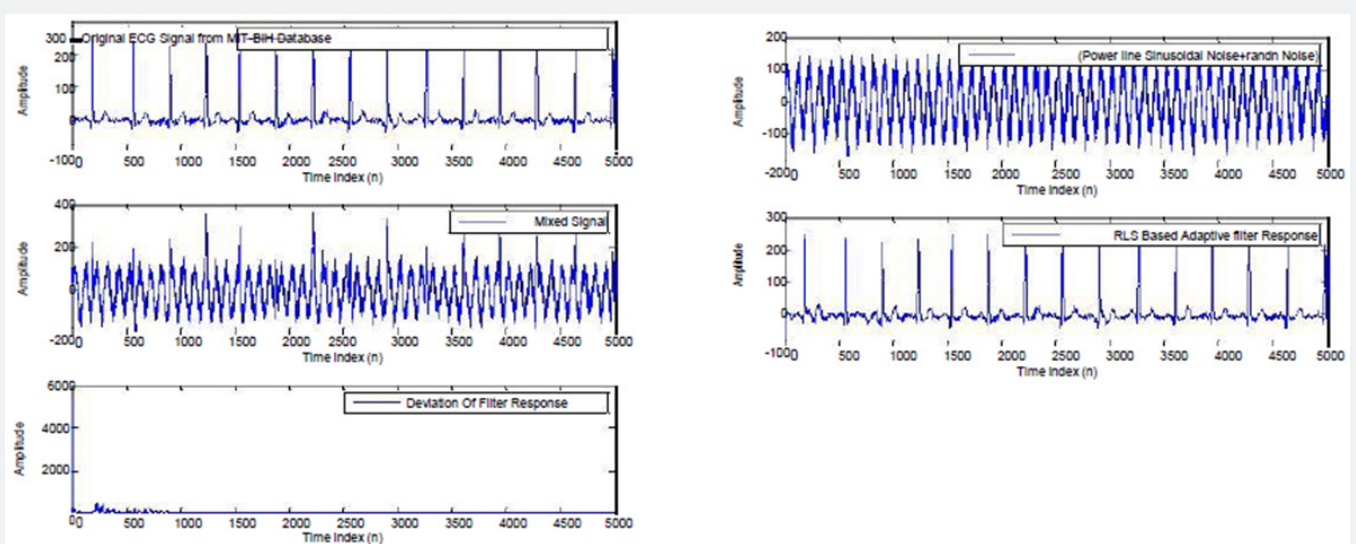

Figure 13: (a) Pure ECG signal taken from MIT-BIH Database (b) $50 \mathrm{~Hz}$ power signal noise with average amplitude $100 \mathrm{mV}$ (c) $50 \mathrm{~Hz}$ power signal noise mixed with pure ECG signal (d) RLS based Adaptive Filter response (e) Square Deviation of RLS based adaptive filter.
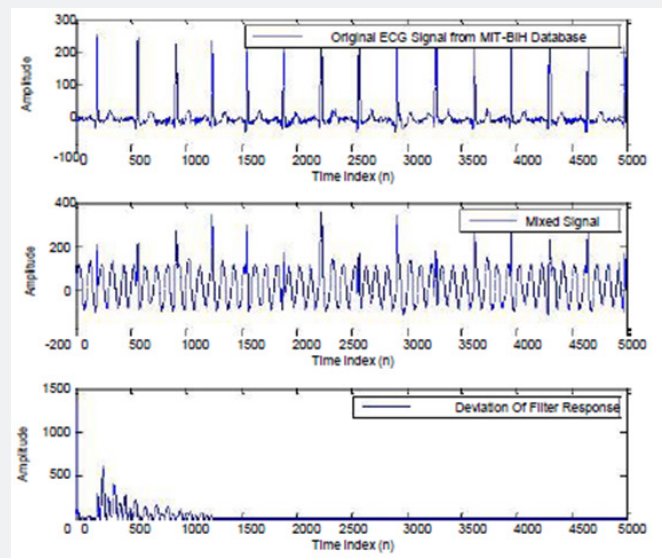
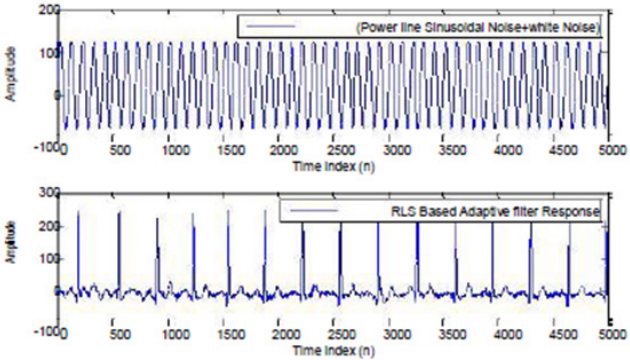

Figure 14: (a) Pure ECG signal taken from MIT-BIH Database (b) $50 \mathrm{~Hz}$ power signal noise with average amplitude $100 \mathrm{mV}+\mathrm{Gaussian}$ Noise with average amplitude $25 \mathrm{mV}$ (c) $50 \mathrm{~Hz}$ power signal noise \& Gaussian Noise are mixed with pure ECG signal (d) RLS based Adaptive Filter response (e) Square Deviation of RLS based adaptive filter.

We have taken 5000 data points of ECG signal from MITBIH database. In our simulation 11data points (from 689 to 699 of original data points of ECG), 201 data points (from 800 to 1000 of original data points of ECG), 300 data points (from 1600 to 1900 of original data points of ECG), 500 data points (from 2000 to 5000) and 6 data points (from 4095 to 5000) are made zero and cubic spline interpolation function was called in MATLAB platform and it could regain the original data points of ECG signal (Figure 15-20). The MATLAB coding result of Spline Interpolation is given below Table 2
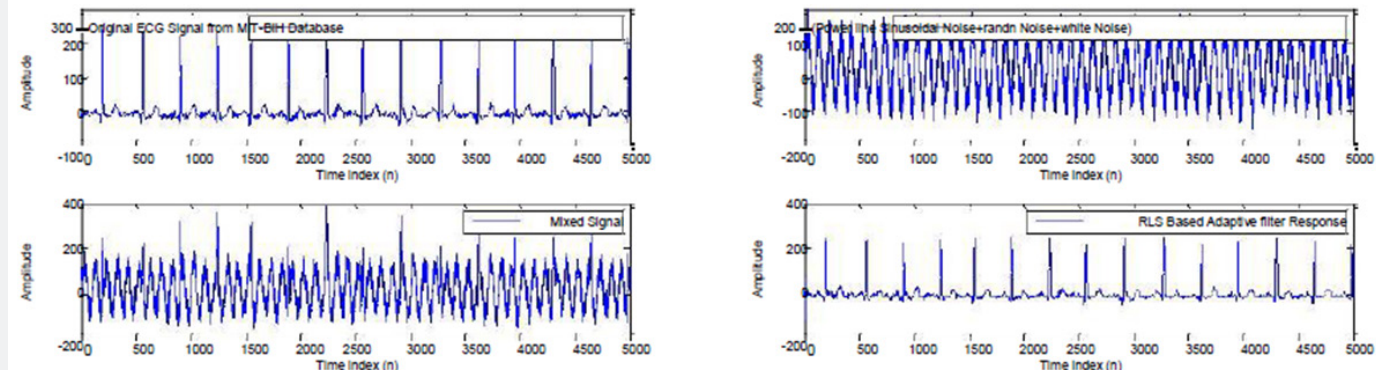

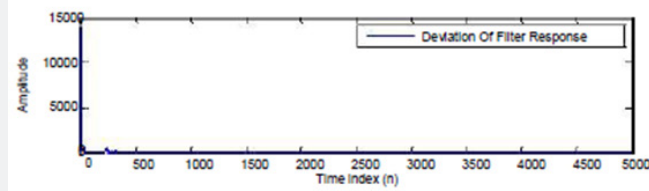

Figure 15: (a) Pure ECG signal taken from MIT-BIH Database (b) $50 \mathrm{~Hz}$ power signal noise with average amplitude $100 \mathrm{mV}+\mathrm{Gaussian}$ Noise with average amplitude $25 \mathrm{mV}+$ Random Noise with average amplitude $25 \mathrm{mV}$ (c) $50 \mathrm{~Hz}$ power signal noise, Gaussian Noise and random Noise mixed with pure ECG signal (d) RLS based Adaptive Filter response (e) Square Deviation of RLS based adaptive filter. 


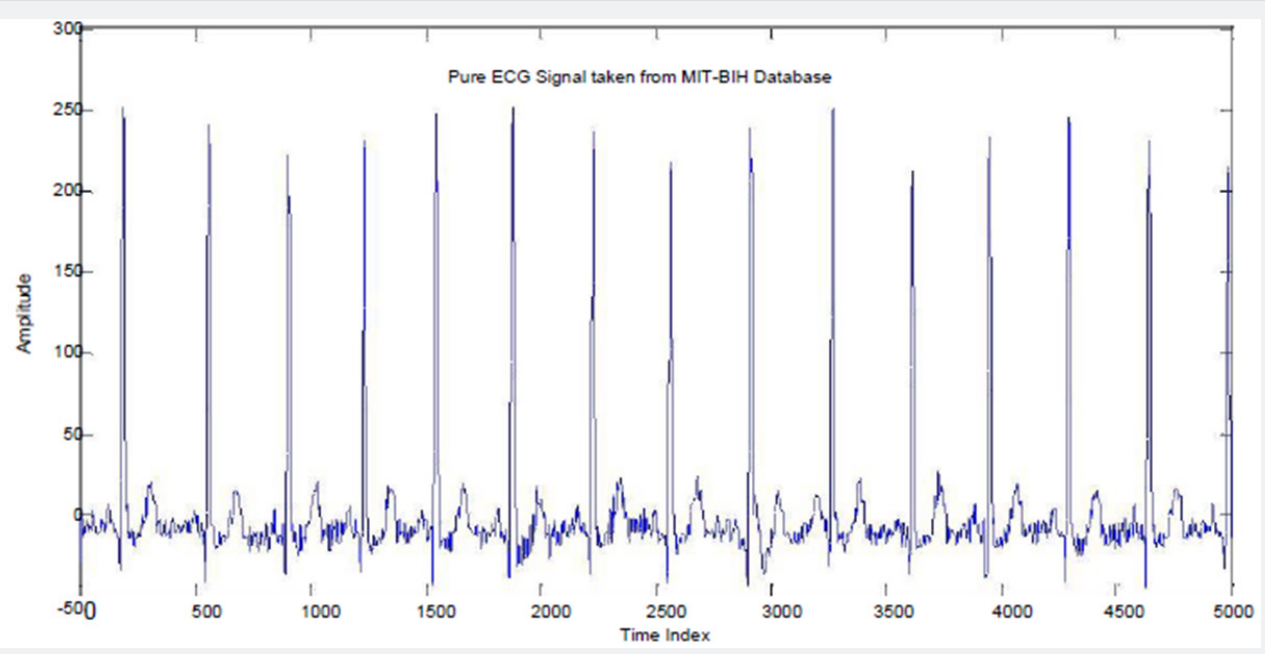

Figure 16: Pure ECG Signal taken from MIT-BIH Database.

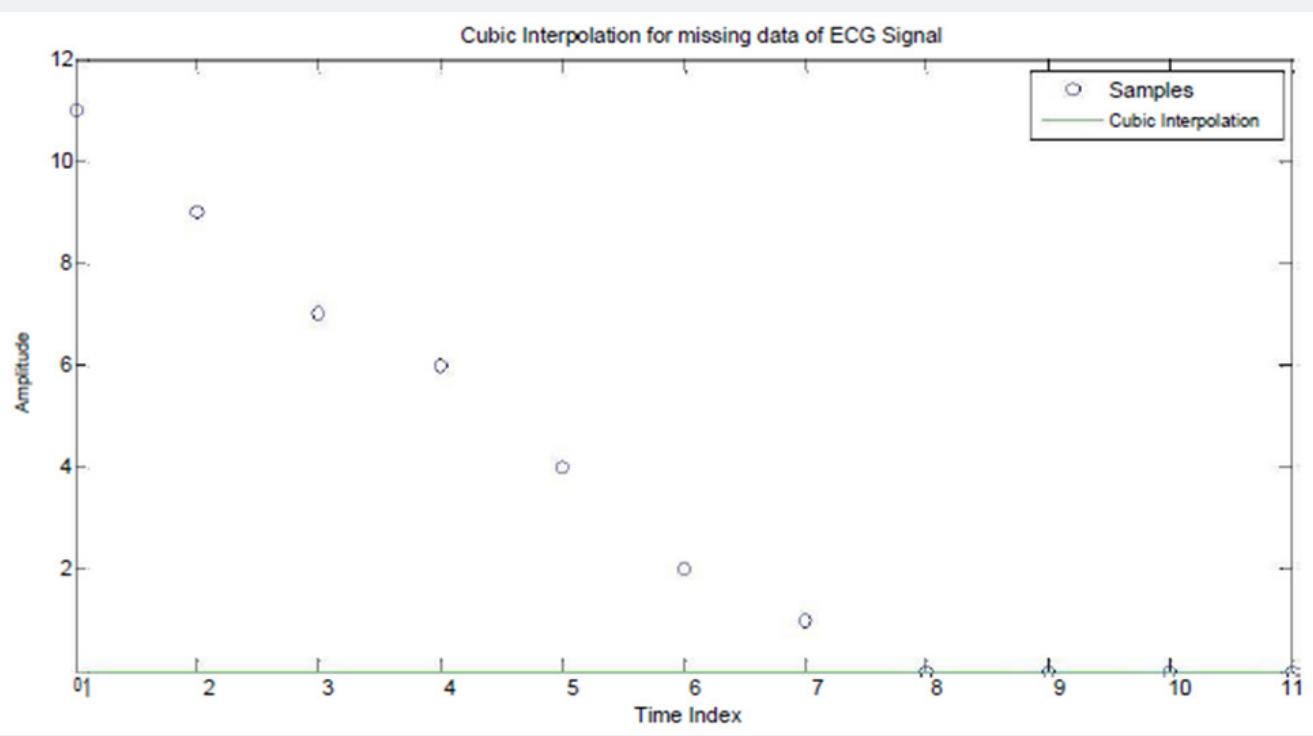

Figure 17: Cubic Spline Interpolation for missing data points of ECG signal from samples 689 to 699 .

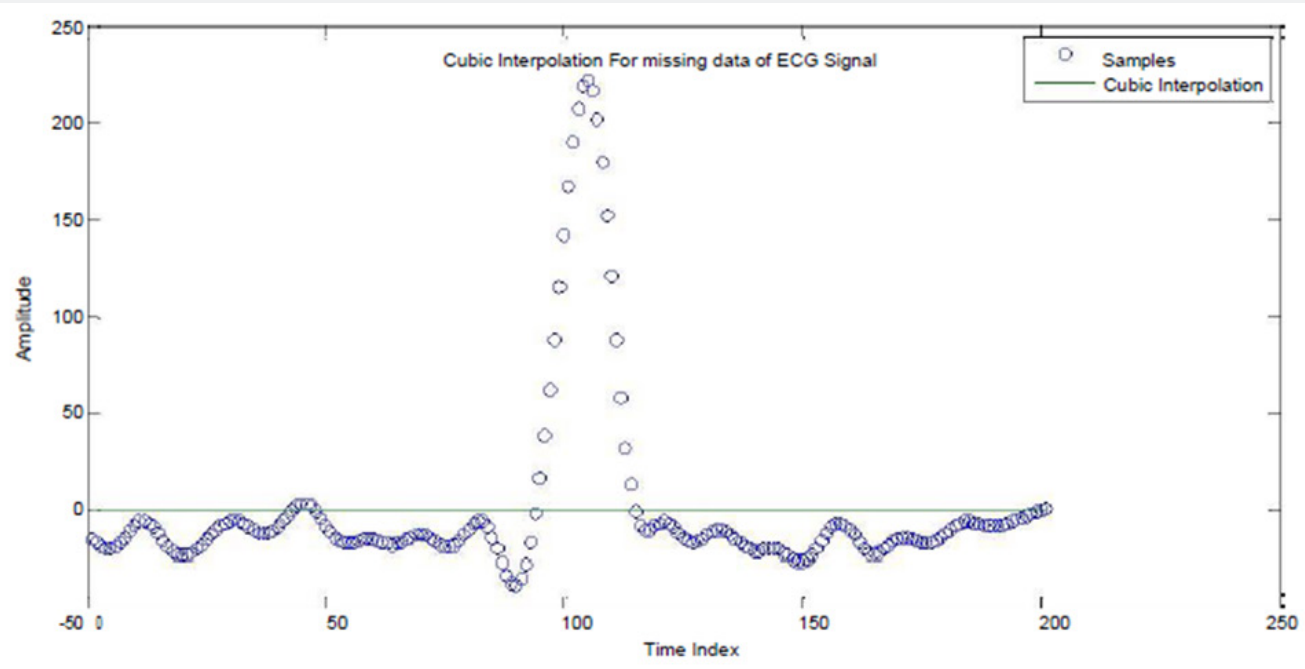

Figure 18: Cubic Spline Interpolation for missing data points (200) of ECG signal from 800:1000 samples. 


\section{Engineering Technology Open Access Journal}

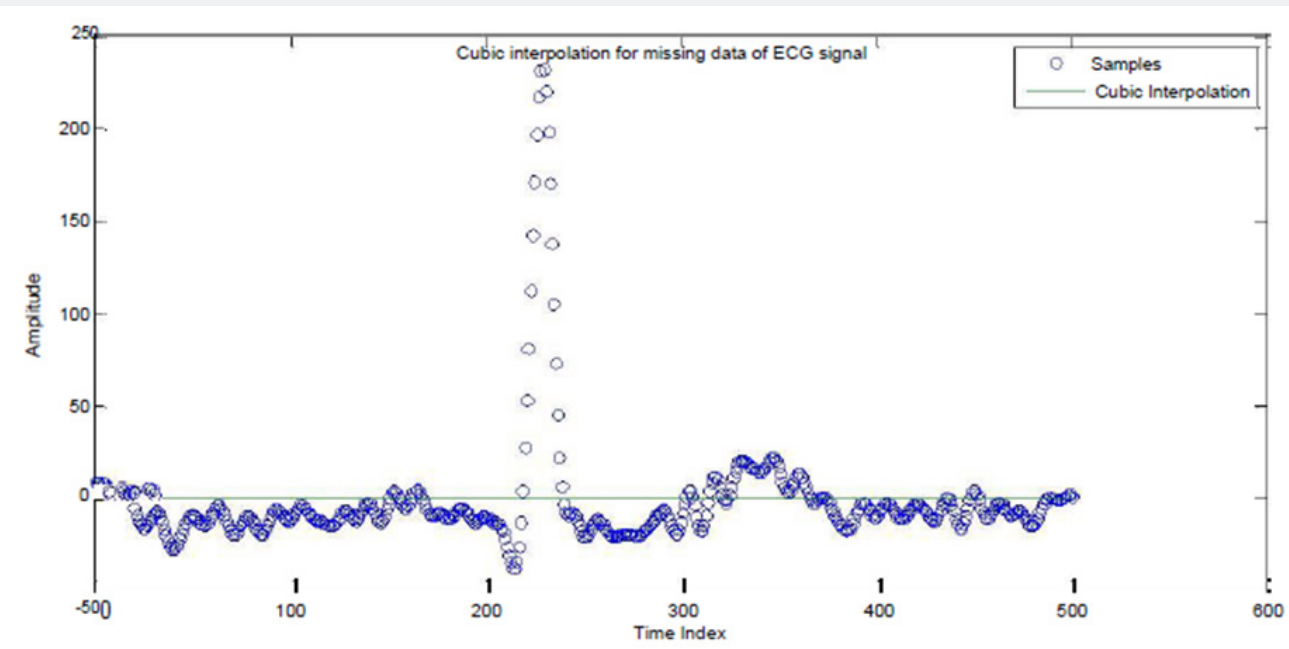

Figure 19: Cubic Spline Interpolation for missing data points (500) of ECG signal from 2000:2500 samples.

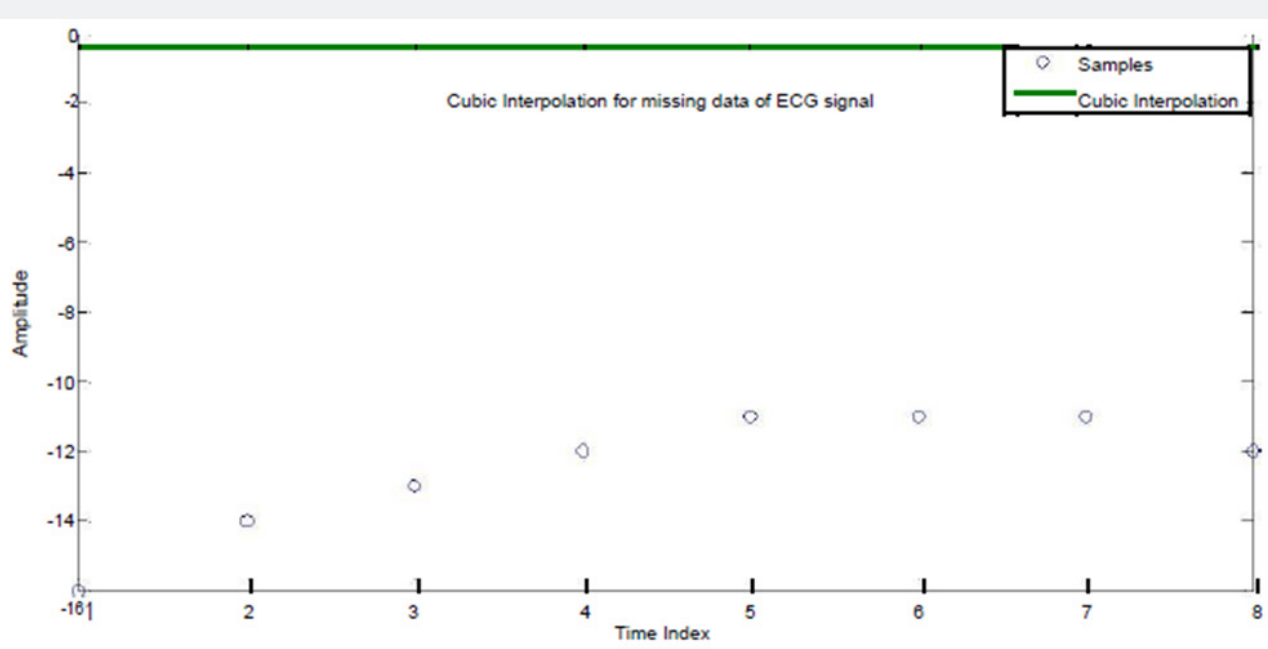

Figure 20: Cubic Spline Interpolation for missing data points (6) of ECG signal from 4095:5000 samples.

The above simulation result suggests that Recursive Least Square algorithm (RLS) performs better than other two Table 1: Normalized Least Mean Square (NLMS) error Calculation.

\begin{tabular}{|c|c|c|c|}
\hline Noise (Amplitude) & $\begin{array}{l}\text { Least Mean } \\
\text { Square(LMS) }\end{array}$ & $\begin{array}{l}\text { Normalized } \\
\text { Least Mean } \\
\text { Square(NLMS) }\end{array}$ & $\begin{array}{l}\text { Recursive } \\
\text { Least } \\
\text { Square(RLS) }\end{array}$ \\
\hline $\begin{array}{c}50 \mathrm{~Hz} \text { power line sinusoidal noise } 100 \mathrm{mV} \text { (40\% of original amplified ECG Signal } \\
\text { Amplitude) }\end{array}$ & $4.61 \times 10-4$ & $8.70 \times 10-5$ & $2.1364 \times 10-5$ \\
\hline $\begin{array}{l}50 \mathrm{~Hz} \text { power line sinusoidal noise } 100 \mathrm{mV} \text { (40\% of original amplified ECG Signal } \\
\text { Amplitude) + Random Noise } 25 \mathrm{mV} \text { (10\% of original amplified ECG Signal Amplitude) }\end{array}$ & $4.57 \times 10-4$ & $1.41 \times 10-4$ & $2.7807 \times 10-5$ \\
\hline $\begin{array}{c}50 \mathrm{~Hz} \text { power line sinusoidal noise } 100 \mathrm{mV}(40 \% \text { of original amplified ECG Signal } \\
\text { Amplitude) + White Gaussian Noise } 25 \mathrm{mV}(10 \% \text { of original amplified ECG Signal } \\
\text { Amplitude })\end{array}$ & $4.66 \times 10-4$ & $8.00 \times 10-5$ & $1.2912 \times 10-4$ \\
\hline $\begin{array}{c}50 \mathrm{~Hz} \text { power line sinusoidal noise } 100 \mathrm{mV} \text { ( } 40 \% \text { of original amplified ECG Signal } \\
\text { Amplitude) + Random Noise \& White Gaussian Noise } 25 \mathrm{mV} \text { (10\% of original amplified ECG } \\
\text { Signal Amplitude })\end{array}$ & $4.94 \times 10-4$ & $1.36 \times 10-4$ & $4.3260 \times 10-5$ \\
\hline
\end{tabular}


Table 2: Normalized Mean Square Error Calculation of Cubic Spline Interpolation.

\begin{tabular}{|c|c|}
\hline $\begin{array}{c}\text { Missing Data Points of ECG } \\
\text { samples }\end{array}$ & $\begin{array}{c}\text { Normalized Mean Square of } \\
\text { Cubic Spline }\end{array}$ \\
\hline $\begin{array}{c}\text { 11 data points(689 to } 699 \\
\text { samples) }\end{array}$ & 0.0909 \\
\hline $\begin{array}{c}\text { 201 data points (800 to } 1000 \\
\text { samples) }\end{array}$ & 0.005 \\
\hline $\begin{array}{c}\text { 300data points (1600 to } 1900 \\
\text { samples) }\end{array}$ & 0.0033 \\
\hline $\begin{array}{c}\text { 500 data points(2000 to } 2500 \\
\text { samples) }\end{array}$ & 0.002 \\
\hline $\begin{array}{c}\text { 6 data points(4095 to } 5000 \\
\text { samples) }\end{array}$ & 0.1667 \\
\hline
\end{tabular}

Normalized mean square error calculation suggests that Cubic Spline performs satisfactorily for regaining missing data points of ECG signal.

\section{Conclusion}

During transmission of ECG signal it may be corrupted due to random noise and Gaussian noise. So we have tested the performances of LMS, NLMS and RLS algorithm of adaptive filter. Our simulation result suggest that RLS could be the best option for recovering ECG signal or denoising EEG signal during transmission through Telemedicine system.

\section{References}

1. Noor K (2010) Comparison of the RLS and LMS Algorithms to Remove Power Line Interference Noise from ECG Signal. Al Khwarizmi Engineering Journal. 6(2): 51-61.

2. Lin YD, Hen $\mathrm{Hu}$ Y (2008) Power Line Interference Detection and Suppression in ECG signal Processing, IEEE Transactions on Biomedical Engineering. 55(1): 354 - 357.

3. Wanl H, Ful R, Shi L (2006) "The Elimination of $50 \mathrm{~Hz}$ Power Line Interference from ECG Using a Variable Step Size LMS Adaptive Filtering Algorithm" Life Science Journal 3(4).



This work is licensed under Creative

Commons Attribution 4.0 License DOI: 10.19080/ETOAJ.2018.01.555555
4. John Mathews V, Scott C. Doughlas, Adaptive Filter, Chapter 4, pp. 1723.

5. Shekh Md, Mahmudul Islam M, Salah Uddin Farid Md, Adnan Kiber Md (2015) Denoising ECG Signal using Adaptive Filter Algorithms and Cubic Spline Interpolation for Regaining Missing data points of ECG in Telecardilogy System, International Journal of Enhanced Research in Science, Technology \& Engineering, ISSN: 2319-7463. 4(12).

6. Luong DT, Thuan ND, Hoang DH (2015) Removal of Power Line Interference from Electrocardiograph (ECG) Using Proposed Adaptive Filter Algorithm, Global Journal of Computer Science and Technology: C Software \& Data Engineering 15(2) Version 1.0.

7. Uzzal Biswas, Maniruzzaman Md Power Line Interference Removal from ECG Signal Using Notch Filter, Adaptive Filter and Wavelet Packet Transform, AIJCSR-362.

8. Sushmitha M, Balaji T (2014) Removing The Power Line Interference from Ecg Signal using Adaptive Filters, IJCSNS International Journal of Computer Science and Network Security 14(11).

9. Neelam, Dahiya R (2014) Analysis of Denoising of Power Line Interference from ECG Signal using Adaptive Algorithm. Progress in Science and Engineering Research Journal 2(4): 086-090.

10. Biswas U, Maniruzzaman Md (2014) Removing Power Line Interference from ECG Signal Using Adaptive Filter and Notch Filter. International Conference on Electrical Engineering and Information \& Communication Technology (ICEEICT), Dhaka, Bangladesh.

11. Maniruzzaman Md, Shimul Billah K Md, Biswas U, Bablu G (2012) Least-Mean-Square Algorithm Based Adaptive Filters for Removing Power Line Interference from ECG Signal, IEEE/OSA/IAPR Interational Conference on [infonnatics, Electronics \& Vision] Dhaka, Bangladesh.

12. Golabbakhsh M, Masoumzadeh MM, Farzan Sabahi M (2011) ECG and power line noise removal from respiratory EMG signal using adaptive filters, Majlesi Journal of Electrical Engineering. 5(4).

13. Morgan D, Kartzer S (1998) On a class computationally efficient rapidly converging generalized NLMS algorithms, IEEE Transaction Education 41: 83.

14. Dhubkarya DC, Aastha K, Raj Kumar (2012) Thenua Simulation of Adaptive Noise Canceller for an ECG signal Analysis, ACEEE Int. J. Signal \& Image Processing 3(01).

\section{Your next submission with Juniper Publishers will reach you the below assets}

- Quality Editorial service

- Swift Peer Review

- Reprints availability

- E-prints Service

- Manuscript Podcast for convenient understanding

- Global attainment for your research

- Manuscript accessibility in different formats

( Pdf, E-pub, Full Text, Audio)

- Unceasing customer service

Track the below URL for one-step submission https://juniperpublishers.com/online-submission.php 